\title{
Comportamento biomecânico das cerâmicas odontológicas: revisão
}

\section{(Biomechanical behavior of dental ceramics: review)}

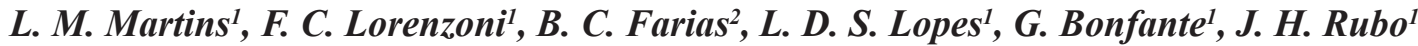 \\ ${ }^{1}$ Faculdade de Odontologia de Bauru, Universidade de S. Paulo \\ ${ }^{2}$ Fundação Bauruense de Estudos Odontológicos \\ Al. Octávio Pinheiro Brisola 9-75, Bauru, SP 17012-901
}

\begin{abstract}
Resumo
Os sistemas cerâmicos representam hoje na odontologia uma alternativa aos metais no tratamento protético. No entanto, esses materiais não atingiram os mesmos resultados a longo prazo da convencional prótese metalocerâmica. Na maioria dos casos, principalmente em próteses anteriores, o comportamento desses materiais é satisfatório. Entretanto, à medida que a demanda por estética exige a utilização de materiais cerâmicos nas regiões posteriores, as taxas de falhas aumentam. Com isso, sabendo o que se pode esperar de cada material é possível não só indicar ou contra-indicar a utilização destas próteses, mas também desenvolver novos materiais e técnicas. O objetivo desta revisão foi apresentar os diversos sistemas cerâmicos e seu comportamento mecânico durante a utilização clínica, relatando as localizações, os tipos e as regiões das falhas mais frequentes nos sistemas atuais, visando à busca por soluções.

Palavras-chave: cerâmica, biomecânica, prótese parcial fixa.
\end{abstract}

Abstract

The current ceramic systems in dentistry represent an alternative to metals in prosthetic treatment. However, these materials did not reach the same long-term results of conventional metal-ceramic prosthesis. In most cases, especially in anterior prostheses, the behavior of these materials is satisfactory. Nevertheless, as the demand for aesthetics requires the use of ceramics in the posterior regions, the rate of failure increases. Therefore, knowing what to expect of each material makes it possible not only to indicate or contraindicate the use of these prostheses, but also to develop new materials and techniques. The objective of this review was to explain the various ceramic systems and their mechanical behavior during the clinical use, reporting the locations, types and regions of most frequent failures in current systems in the search for solutions.

Keywords: ceramics, biomechanics, denture partial fixed.

\section{INTRODUÇÃO}

\section{Sistemas cerâmicos}

A estética é um fator primordial na Odontologia. As restaurações ceramo-cerâmicas, por sua vez, em razão de sua alta capacidade de mimetizar os tecidos dentais, são a escolha de preferência de muitos profissionais e pacientes; contudo, ocasionalmente estas coroas falham gerando desconforto e inconvenientemente abrem questões sobre a efetividade desse tipo de tratamento.

Definir a efetividade e determinar a média de longevidade é o principal objetivo dos estudos de sobrevida em prótese parcial fixa (PPF). Tais dados trazem informações importantes sobre o prognóstico da terapia protética e dos materiais utilizados, sendo possível monitorar o modelo estudado e ranquear as possíveis causas de falhas.

As cerâmicas possuem excelentes características, tais como: biocompatibilidade, estabilidade de cor, baixa condução térmica, baixo acúmulo de placa, resistência à abrasão, além de promover uma excelente estética [1]. Entretanto, as cerâmicas são frágeis e de baixa resistência mecânica, quando submetidas às tensões de tração, o que compromete seu desempenho clínico em alguns aspectos. As tradicionais coroas metalocerâmicas consistem numa infraestrutura de metal recoberta por porcelana. A infraestrutura de metal é opaca e por consequência não consegue imitar a translucidez do dente natural. Os sistemas totalmente cerâmicos surgiram com o intuito de eliminar estas infraestruturas, na tentativa de promover uma melhor distribuição da reflexão da luz, resultando assim, numa melhor estética. Estes sistemas podem ser fabricados em uma única camada (coroas monolíticas), como as vitrocerâmicas (p. ex. cerâmicas à base de dissilicato de lítio ou de leucita), e posteriormente pintada para permitir as características de cor ou podem ser confeccionadas em camadas composta pela infraestrutura cerâmica e pela cerâmica de cobertura.

Atualmente existe uma grande variedade de classes cerâmicas disponíveis para distintas indicações, de acordo com seus fabricantes. Contudo, não existe um único sistema totalmente cerâmico passível de ser empregado em todas as situações clínicas. Em pouco tempo, impulsionadas por uma demanda estética crescente, cerâmicas para infraestruturas foram desenvolvidas e podem ser indicadas 
com certa segurança em situações clínicas específicas. O aumento crescente na resistência destes materiais permitiu a substituição de molares, os quais estão na região com maior esforço oclusal.

O sistema DICOR (não mais disponível no mercado) era uma vitrocerâmica com resistência à fratura média de $70 \mathrm{MPa}$ [2]. A incorporação do óxido de alumínio permitiu um aumento de 4 a 6 vezes em relação a DICOR, sendo que representantes deste material, como o In-Ceram Alumina e o Procera, apresentam resistência média de $550 \mathrm{MPa}$ [3] e $472 \mathrm{MPa}$ [4], respectivamente. Entretanto, a zircônia é o material cerâmico com maior resistência, cerca de $1150 \mathrm{MPa}$ [5]. Muito do entusiasmo observado principalmente com a zircônia deve-se ao fato de que sua resistência aproximase à encontrada em algumas ligas metálicas [6]. Entretanto, a resistência à fratura por si só não é capaz de predizer o comportamento em longo prazo, devendo considerar outras propriedades mecânicas, como a tenacidade à fratura, dureza e módulo de elasticidade. Os componentes biológicos (oclusão, atividade funcional e parafuncional), além do tipo de substrato (dentina, esmalte, resina composta, liga metálica), espessura da linha de cimentação, tipo de cimento e adesão da cerâmica ao substrato dentário, representam tópicos importantes que devem ser considerados durante a seleção de um sistema. Adicionalmente, os resultados iniciais dos sistemas totalmente cerâmicos tem se apresentado menos favoráveis mecanicamente quando comparados às tradicionais coroas metalocerâmicas [1].

Uma das principais características dos sistemas cerâmicos relaciona-se com a translucidez da infraestrutura [7]. Quanto mais translúcido for o sistema, mais apropriada será sua indicação para a solução de casos com extrema exigência estética. Contudo, translucidez e resistência são grandezas inversamente proporcionais. $\mathrm{O}$ aumento do conteúdo cristalino na composição das cerâmicas, com diminuição da fase vítrea, aumentou os valores de resistência à fratura, por outro lado, estes sistemas são mais opacos ou menos translúcidos [7-9]. A translucidez de vários sistemas cerâmicos foi avaliada, tendo como grupos controles uma porcelana de cobertura e uma liga metálica, com espessuras compatíveis com as indicadas para a clínica (Fig. 1).

Neste estudo, foi indicado que os sistemas IPS Empress $(0,5 \mathrm{~mm})$ e In-Ceram Spinell foram os mais translúcidos, porém, estes foram mais opacos do que a cerâmica Vitadur Alpha (controle). Adicionalmente, foi verificado que não houve diferença estatística entre os sistemas IPS Empress 2 (com 0,8 mm de espessura) com o Procera AllCeram e foi apontado que os valores da translucidez do metal (grupo controle) e da zircônia foram similares estatisticamente [10]. Apesar da baixa translucidez da zircônia, similar às das ligas metálicas, aquela não apresenta substrato acinzentado, assim, não há a necessidade de técnica adicional para mascarar a cor da infraestrutura.

Taxas de insucesso elevadas apresentadas pelas próteses totalmente cerâmicas levaram à necessidade de evolução desses materiais. Melhorias nas propriedades mecânicas das cerâmicas foram alcançadas pelo aprimoramento do método

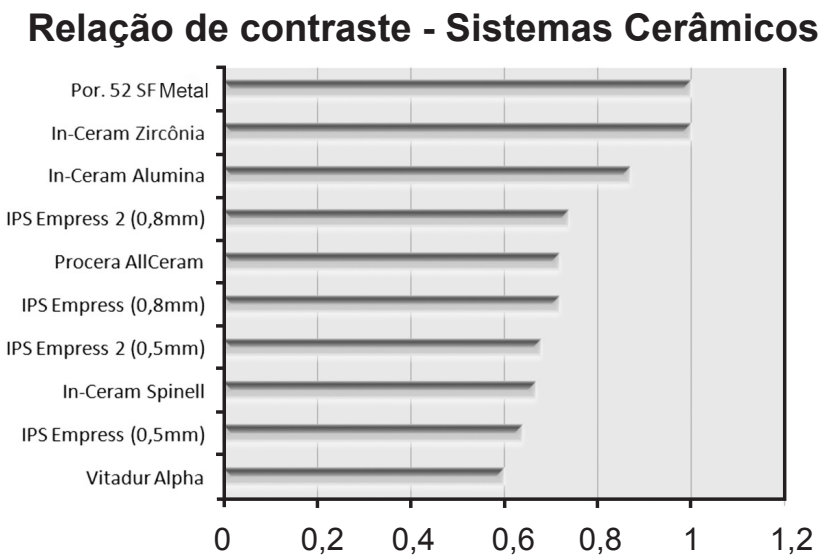

Figura 1: Valores médios da relação de contraste dos principais sistemas cerâmicos. Notar que quanto mais próximo do valor 0 (zero), mais translúcido é o material. Adaptado de [10].

[Figure 1: Mean values of the contrast ratio of the main ceramic systems. Note that material with the value closer to 0 (zero) is more translucent. Adapted from [10].]

de processamento e pela introdução de maiores frações e de novas fases cristalinas. Atualmente, diversos sistemas cerâmicos estão presentes no mercado odontológico, como as cerâmicas à base de sílica (porcelanas e vitrocerâmicas a base de leucita e de dissilicato de lítio) e à base de óxido (alumina, espinélio e zircônia estabilizada por ítria). Esses reforços introduzidos à cerâmica apresentam características específicas na busca do aumento da tenacidade.

\section{Cerâmicas à base de sílica}

Para reforçar as cerâmicas à base de sílica, buscouse aumentar a fração da fase cristalina, proporcionando melhorias significativas nas propriedades mecânicas por meio do aumento do módulo de elasticidade e consequente limitação na propagação de trincas $[6,11]$. Observa-se que muitos outros mecanismos que atuam sinergicamente, embora com efetividades distintas, têm sido empregados para melhorar as propriedades mecânicas das cerâmicas, destacando o mecanismo de deflexão das trincas [12]. As vitrocerâmicas com reforço de leucita trouxeram um ganho na resistência flexural na ordem de $35-55 \%$ em relação às porcelanas feldspáticas. Já as cerâmicas reforçadas por dissilicato de lítio são cerca de 4 vezes mais resistentes do que as feldspáticas.

Durante a mastigação desenvolvem-se forças de grande intensidade, $69 \mathrm{~N}$ na região anterior e $516 \mathrm{~N}$ na região posterior [13]. Além disso, cargas compressivas de até 838 $\mathrm{N}$ foram encontradas em pacientes com sinais de atividade parafuncional (bruxismo) [14]. Tudo isso associado a um ambiente úmido (saliva) e com constantes variações de temperatura e $\mathrm{pH}$. Sendo assim, mesmo com um significativo aumento da resistência flexural de $120 \mathrm{MPa}$ e tenacidade de $1,2 \mathrm{MPa} / \mathrm{m}^{1 / 2}$ para a vitrocerâmica com partículas de leucita e $400 \mathrm{MPa}$ e $3,5 \mathrm{MPa} / \mathrm{m}^{1 / 2}$ para a vitrocerâmica com fibras 
curtas de dissilicato de lítio, o uso destes é restrito [15]. Vale ressaltar que a leucita foi empregada na odontologia no início dos anos $60 \mathrm{sem}$ a intenção de prover qualquer reforço à porcelana de cobertura, mas com a proposta de compatibilizar o coeficiente de expansão térmica entre esta porcelana com as ligas metálicas utilizadas para confeccionar a infraestrutura na época [16]. Recentemente a leucita foi empregada como fase de reforço [17] e dessa forma sua distribuição na matriz vítrea tornou-se mais homogênea, com grãos de leucita com aproximadamente 1,7 $\mu \mathrm{m}$ de diâmetro [18], o que permitiu o seu emprego como cerâmica de infraestrutura. Representantes desses materiais são IPS-Empress 1 e 2 (Ivoclar-Vivadent), com leucita e dissilicato de lítio, respectivamente, os quais apresentaram um bom resultado estético para restauração parciais (faceta, inlay e onlay) e coroas e próteses parciais fixas (PPF) de 3 elementos até pré-molar [19]. O método de processamento do sistema Empress é por meio da técnica da cera perdida. Assim, a moldagem pela injeção a quente sob pressão ("heat pressing"), utiliza um padrão em cera de infraestrutura ou da coroa a ser produzida, a qual é incluída em um molde refratário. Este refratário é inserido no interior de um forno convencional para eliminar a cera, pré-aquecido a $700{ }^{\circ} \mathrm{C}$, durante $30 \mathrm{~min}$. Dessa forma, cria-se um espaço para o seu preenchimento subsequente com a vitrocerâmica. Ainda neste forno, a pastilha de cerâmica, que pode ser reforçada tanto pela leucita quanto pelo dissilicato de lítio, é posicionada na abertura do refratário, juntamente com o cursor de alumina. Este conjunto, (refratário, cerâmica, cursor em alumina) é inserido no interior do forno desenvolvido para a técnica, o qual introduz a cerâmica por meio de fluxo viscoso. A temperatura inicial é de $700{ }^{\circ} \mathrm{C}$, com taxa de aquecimento de $60{ }^{\circ} \mathrm{C} / \mathrm{min}$, com temperatura final para a injeção da cerâmica de $920{ }^{\circ} \mathrm{C}$ (para o dissilicato de lítio), mantendo tempo de injeção por 20 min à pressão de 5 bar. A temperatura final para a técnica com injeção de cerâmica com leucita é de $1180{ }^{\circ} \mathrm{C}[20]$.

Contudo, a resistência dos materiais cerâmicos é limitada pela presença e distribuição dos defeitos estruturais ou das falhas em quantidades e tamanhos críticos [21]. O fenômeno da corrosão sob tensão (slow crack growth) provoca a degradação das propriedades mecânicas das cerâmicas, tornando a falha dependente do tempo [22], diminuindo a sobrevida. Entretanto, em cerâmicas que não apresentam o fenômeno da transformação de fase para sua tenacificação, a deflexão da trinca é considerada o principal mecanismo envolvido para o aumento da resistência à propagação da trinca, por meio da formação de anteparos (fase cristalina) na ponta da trinca, diminuindo o fator de intensidade de tensão na ponta da trinca, resultando no aumento da tenacidade e do limite de fadiga da cerâmica [23]. Assim, tensões abaixo do limite de fadiga não são suficientes para a propagação da trinca.

O ambiente oral influencia sobremaneira este processo de corrosão, pois apresenta tensão (estresse) mastigatória, água proveniente da saliva e dos túbulos dentinários, variações de temperatura e de $\mathrm{pH}$, além da associação de diferentes coeficientes de expansão térmica entre materiais diferentes [24]. Portanto, sob carga, a trinca pode propagar-se de um defeito e crescer lentamente em um estágio inicial, com posterior aceleração da velocidade de propagação da trinca até atingir um tamanho crítico, culminando na falha [25, 26].

As propriedades mecânicas dos materiais cerâmicos devem ser aprimoradas para que se possa aproximar as taxas de sucesso dos sistemas totalmente cerâmicos com as do metalocerâmico [27]. Metodologias têm sido utilizadas para aumentar a resistência das cerâmicas, como a incorporação de um substrato metálico com um coeficiente de expansão térmico maior do que a porcelana de cobertura, criando uma força residual compressiva nesta cerâmica, o glazeamento com a intenção de formar uma película vítrea minimizando a influência da trincas, assim como a utilização de uma fina camada de glaze com um coeficiente de expansão térmico menor do que a porcelana subjacente para criar nesta uma força residual compressiva [28]. Entretanto, métodos como o tratamento de têmpera e a troca iônica [29] podem ser utilizados para criar uma zona compressiva na superfície da cerâmica que irá opor-se às forças de carregamento (forças trativas), diminuindo o fenômeno da corrosão sob tensão (slow crack growth), assim como a velocidade de propagação da trinca [27].

\section{Cerâmicas a base de óxido}

Cerâmicas com alto teor de alumina: as cerâmicas baseadas em alumina foram desenvolvidas para Odontologia em 1965. Seguindo os mesmos conceitos do aumento da rigidez e de deflexão da trinca, utiliza óxidos cerâmicos como "limitadores da propagação de trincas" [30]. A primeira tentativa de introdução de alumina foi limitada a $50 \%$ de óxido a porcelana, mas foi aperfeiçoada aumentando a capacidade de adição dos óxidos para aproximadamente 85\% em volume (In-Ceram, Vita Zhranfabrik), através do método conhecido como colagem de barbotina (slip-casting), o qual aumentou a resistência das porcelanas à base de alumina. Neste sistema, o primeiro passo é formar uma estrutura porosa de alumina, ainda frágil do ponto de vista mecânico, que posteriormente é infiltrada por vidro de baixa viscosidade que proporciona à cerâmica elevada densidade e resistência. Já o sistema Procera (Nobel Biocare) por meio da prensagem a seco do pó de alumina no troquel, obteve $99,9 \%$ de óxidos. Atualmente os sistemas CAD/CAM (Computer Assisted Design/ Computer Assisted Machining) com scanners para captura de imagens sem contato e em três dimensões, transformam o sistema em um processo preciso e confiável. O sistema Vita In-Ceram AL2000 Cubes utiliza blocos présinterizados em alumina para construir coroas ou pontes fixas por meio da tecnologia CAD/CAM. O sistema Cerec InLab pode ser o recurso utilizado para capturar a imagem através do escaneamento óptico do preparo dentário e para usinar a peça protética. Após a usinagem da infraestrutura, esta é sinterizada em um forno específico. O ciclo térmico inicia-se com uma hora de aquecimento até atingir a temperatura final de $1530{ }^{\circ} \mathrm{C}$, a qual é mantida por $2 \mathrm{~h}$. Após a sinterização, 
ocorre o resfriamento da peça dentro do forno até atingir $400{ }^{\circ} \mathrm{C}$. O ciclo térmico total leva cerca de $8 \mathrm{~h}$. Todos esses avanços no processamento resultaram em um material com alto conteúdo de alumina densamente sinterizado e com porosidade reduzida que é capaz de suportar cargas flexionais de $699 \mathrm{MPa}$ e que possui uma tenacidade de 3,1 a 4,6 MPa/ $\mathrm{m}^{1 / 2}$, ampliando a indicação das restaurações totalmente cerâmicas também para a região posterior [31]. Baseado no desenvolvimento da nova geração de compósitos com matriz cerâmica, as aluminas infiltradas por vidro receberam um acréscimo de $35 \%$ de zircônia parcialmente estabilizada elevando a resistência do material para $800 \mathrm{MPa}$ e sua tenacidade à fratura para $6-8 \mathrm{MPa} / \mathrm{m}^{1 / 2}[15]$. Estes resultados demonstram o grande potencial odontológico dos materiais à base de zircônia.

Zircônia tetragonal policristalina estabilizada com ítria (Y-TZP): A zircônia possui um mecanismo para aumento da tenacidade que a difere das demais cerâmicas utilizadas em Odontologia, a transformação de fases. O aumento de tenacidade por transformação baseia-se na obstrução da propagação da trinca por meio da zircônia estabilizada normalmente com 3\% em mol de óxido de ítrio. Esta estabilização permite que a zircônia, que em temperatura ambiente estaria na fase monoclínica (estável), se mantenha na fase tetragonal [32]. O processo de obstrução da propagação da trinca ocorre na presença de campos de tensões de tração na ponta da trinca (surgem quando o material é submetido a uma tensão externa), que induz a desestabilização das partículas tetragonais e a ocorrência de uma transformação de fase displaciva (martensítica) para monoclínica. Esta transformação é acompanhada por um aumento de volume (3-5\%) do material, que provoca uma força compressiva contra as superfícies da trinca, fechando-a e dificultando sua propagação ou crescimento $[15,33]$. Por meio deste fenômeno, a zircônia Y-TZP atinge resistência à flexão de 900-1200 MPa e valores de tenacidade de 6-10 $\mathrm{MPa} / \mathrm{m}^{1 / 2}$. Com propriedades mecânicas superiores às demais cerâmicas, a zircônia Y-TZP ampliou as indicações das próteses cerâmicas para próteses parciais fixas de $3 \mathrm{a}$ 4 elementos em qualquer região bucal [15]. Como visto, a zircônia apresenta excelentes características mecânicas, como a tenacidade à fratura e a resistência flexural, em parte devido a transformação da fase metaestável tetragonal para a monoclínica [34]. Entretanto, a zircônia apresenta o problema relacionado com sua degradação por envelhecimento a baixas temperaturas [35], sendo que este fenômeno ocorre na superfície da zircônia e degrada suas excelentes propriedades mecânicas. A zircônia quer seja estabilizada por óxido de ítrio, cério, cálcio ou magnésio é susceptível a degradação em várias situações de ambientes, como ao vapor d'água, à umidade do ar, e a outros fluidos; contudo, em ambiente aquoso o efeito é mais catastrófico e ocorre em curtos períodos de tempo [34]. É importante salientar que próteses fixas ou unitárias podem ser cimentadas sobre pilares vitais, os quais apresentam umidade oriunda dos túbulos dentinários, sendo aqui importante a capacidade do cimento de impedir que a superfície da zircônia entre em contato com esta umidade. Por meio do mesmo mecanismo (transformação da fase tetragonal-monoclínica) que pode beneficiar a Y-TZP melhorando suas propriedades mecânicas [36], pode-se deteriorar estas mesmas propriedades mecânicas através do processo de envelhecimento [34]. Assim, a degradação é causada por esta transformação de fase, a qual é acompanhada pelo surgimento de micro ou macro trincas, ocorrendo primeiramente na superfície do material, sendo que a água ou o vapor d'água pode atuar sinergicamente neste mecanismo de envelhecimento [37]. Dessa forma, este processo ocorre primeiramente em grãos localizados, na superfície, com uma cascata posterior de enventos que culmina na formação de microtrincas e tensões nos grãos vizinhos, favorecendo a penetração de àgua e promovendo a transformação de fase em um maior número de grão. Finalmente ocorre a formação de uma superfície rugosa associada a microtrincas mais extensas [32]. As consequências deste envelhecimento em odontologia ainda não é um tópico de interesse, pois estudos apontam uma quantidade surpreendente de falhas coesivas na porcelana de revestimento, raramente expondo a infraestrutura, de $6 \%$ [38] e 15,2\% [31] num período de 3 a 5 anos. Por outro lado, em ortopedia é um tópico controverso, pois se relaciona os insucessos (fraturas) de próteses de cabeça de fêmur ao envelhecimento da zircônia [32]. Este fato pode ser explicado ao tempo de uso deste biomaterial na medicina superior a 20 anos, contra a sua incipiente utilização em larga escala na odontologia.

Contudo, o tipo de fatura das cerâmicas à base de silicato e as com alto teor de alumina, assim como as cerâmicas a base de zircônia e todas as cerâmicas odontológicas atuais, é similar. Todas não sofrem deformação plástica antes da propagação da trinca e a ruptura do material é denominada de fratura frágil [39]. Este tipo de fratura catastrófica impossibilita reparos (dano irreversível), obrigando a substituição da peça, muitas vezes precocemente.

\section{Longevidade}

Vários fatores governam a longevidade das próteses. As falhas podem ser classificadas em biológicas, mecânicas e relacionadas ao paciente. Entretanto, há discordâncias sobre a definição do termo "falha", o que pode trazer dificuldade durante a análise e a interpretação dos dados.

\section{Complicações biológicas}

As complicações biológicas são menos frequentes do que as complicações mecânicas em coroas ou PPF totalmente cerâmica [40]. A falha biológica mais frequente é a perda de vitalidade pulpar, com taxa de $2,1 \%$ em 5 anos [40]. A perda da vitalidade pulpar ou fratura do pilar pode estar relacionada com o desgaste excessivo da estrutura dentária resultado de preparos dentários extensivos [41]. A segunda maior causa de fracassos em sistemas totalmente cerâmicos está relacionada com a recidiva de cárie, com taxa anual de 
0,37\% [40]. Já no sistema metalocerâmico, as complicações biológicas são as mais representativas. Em uma análise retrospectiva de 332 PPF em 20 anos foi observado que a razão mais comum para complicações irreversíveis foi a recidiva de cárie $(22 \%)$ seguida pela perda de retenção $(15,3 \%)$ [42].

\section{Complicações mecânicas}

Como dito, as cerâmicas são frágeis [43] e suscetíveis à fratura, especialmente quando sujeitas a carregamento cíclico e em ambiente úmido [44]. O desempenho estrutural dos sistemas cerâmicos permanece menos estável do que o do sistema metalocerâmico, pois a fratura catastrófica ou da porcelana de revestimento afeta de 5 a $10 \%$ das coroas totalmente cerâmicas em um período de 6 anos [45]. A evidência clínica indica que a maioria das fraturas que ocorre em estruturas protéticas é o resultado da falha por fadiga, a qual é explicada pelo desenvolvimento e a propagação de trincas microscópicas em áreas de concentração de tensão, sendo a falha catastrófica o resultado final do carregamento cíclico que excede a capacidade mecânica do material [46]. A capacidade de resistência do material à propagação de trincas pode ser descrita pela propriedade de tenacidade à fratura $\left(K_{\mathrm{IC}}\right)$, a qual é uma propriedade intrínseca do material, sendo um importante parâmetro a ser avaliado durante a seleção do sistema cerâmico [47]. As trincas podem ter origem em locais que envolvem defeitos de processamento, como: aspereza superficial, porosidades e inclusão de impurezas; e de defeitos inerentes ao material, também relacionados ao tamanho da partícula, à tensão residual e à presença de micro-trincas [48]. A correlação entre os resultados in vitro com os achados clínicos e de estudos longitudinais é necessária para que se possa entender o processo de fratura e para propor soluções para os problemas evidenciados em clínica. Investigações em testes laboratoriais estabeleceram condições críticas limite, que produzem dados que são utilizados para propor soluções aos problemas clínicos dos sistemas cerâmicos, o que em última análise se traduz em maior longevidade. Estudos prévios qualificaram e quantificaram as várias formas de danos que podem ser provocados nos materiais cerâmicos e que podem apresentar potencial em originar fraturas. Existem dois modos básicos de falhas, um sob o ponto que recebe a carga, com origem na superfície oclusal, denominado de trinca tipo cone (interno ou externo) e outro na face oposta ao do ponto da carga, onde trincas se originam na superfície de cimentação das infraestruturas cerâmicas ou na interface entre a porcelana de cobertura e a infraestrutura, denominada trinca tipo radial [49], Fig. 2.

A trinca cônica externa (trinca de contato Hertziano), muito embora seja o primeiro tipo de dano a surgir durante a carga cíclica e de forma precoce, apresenta angulação de propagação de aproximadamente $22^{\circ} \mathrm{em}$ relação à superfície oclusal, porém raramente apresenta expressão clínica. A trinca cônica interna é potencialmente mais agressiva, pois pode propagar em direção à interface porcelana de

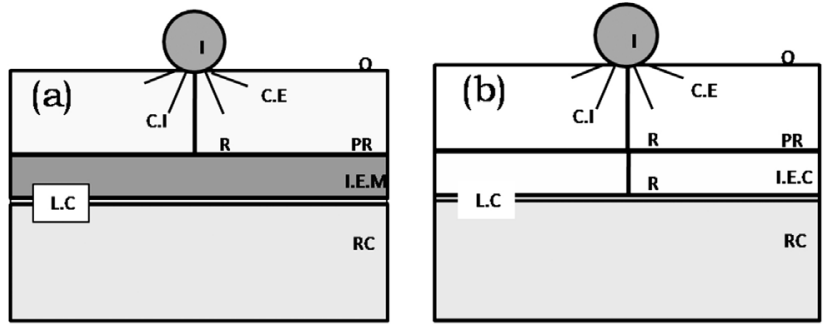

Figura 2: Diagrama esquemático da geometria da trinca, após carregamento cíclico com indentador esférico, em uma estrutura metalocerâmica (a) e em uma ceramo-cerâmica (b). Indentador metálico (I), superfície oclusal (O), cone externo $(\mathrm{CE})$, cone interno $(\mathrm{CI})$, trinca radial $(\mathrm{R})$, porcelana de revestimento (PR), infraestrutura metálica (I.E.M), infraestrutura cerâmica (I.E.C), linha de cimentação (LC), substrato em resina composta (RC). Adaptado de [51]. [Figure 2: Schematic diagram of geometry of the crack after cyclic loading with a spherical indenter in a metal-ceramic (a) and in a ceramo-ceramic (b) system. Metallic indenter (I), occlusal surface (O), outer cone (EC), inner cone (CI), radial cracks $(R)$, porcelain veneer $(P R)$, metal framework (IEM), ceramic framework (IEC) cementation line (LC), composite resin substrate (RC). Adapted from [51].]

revestimentolinfraestrutura cerâmica, sendo que apresenta uma angulação maior do que a do cone externo, podendo provocar a fratura coesiva da porcelana de revestimento [50]. A trinca tipo radial é considerada a mais relevante e considerada como falha do sistema, pois pode provocar a fratura catastrófica de toda a restauração $[49,51]$. A trinca radial pode apresentar-se de duas maneiras distintas. Quando a trinca radial origina-se da superfície de cimentação da infraestrutura cerâmica, esta pode propagar-se em direção à porcelana de revestimento resultando na fratura catastrófica; quando a trinca radial origina-se da interface porcelana de revestimentolinfraestrutura cerâmica, esta pode propagarse através da porcelana de revestimento, apresentando como expressão clínica a fratura coesiva da porcelana de revestimento.

Coroas totalmente cerâmicas apresentaram como principal causa das complicações mecânicas a fratura catastrófica, cerca de $85 \%$ de todas as complicações; ressalta-se que este estudo não avaliou coroas à base de zircônia. Assim, como as trincas radiais são as responsáveis por fraturas catastróficas, pode-se argumentar que este tipo de trinca é a principal causa de falhas em coroas totalmente cerâmicas [40].

Um grande número de fatores pode afetar o desempenho das coroas totalmente cerâmicas, os quais podem ser controlados pelo clínico ou são dependentes do paciente [52]. O desafio é entender como cada fator ou a interação entre estes podem influenciar a longevidade clínica. Um fator em especial chama a atenção. A análise dos trabalhos de acompanhamento clínico demonstra claramente a preocupação dos autores em excluir pacientes com atividade parafuncional, pois podem produzir forças oclusais com 
potencial deletério sobre os sistemas totalmente cerâmicos $[53,54]$.

Quando os valores das taxas de sobrevida dos sistemas ceramo-cerâmicos são agrupados de acordo com a posição na boca, torna-se evidente que as próteses localizadas na região anterior apresentam sobrevida superior às localizadas na região posterior [40]. Contudo, observa-se uma maior incidência de substituição para as coroas metalocerâmicas na região anterior em detrimento de necessidades estéticas, pois frequentemente a cinta metálica e o escurecimento da região marginal afetam a estética da região cervical da coroa resultando em uma aparência não natural [55].

A resistência à fratura das próteses parciais fixas (PPFs) está relacionada com a forma, o tamanho e a posição do conector, além do comprimento do(s) pôntico(s) [56], Fig. 3. A forma mais comum de falhas em PPFs totalmente cerâmicas

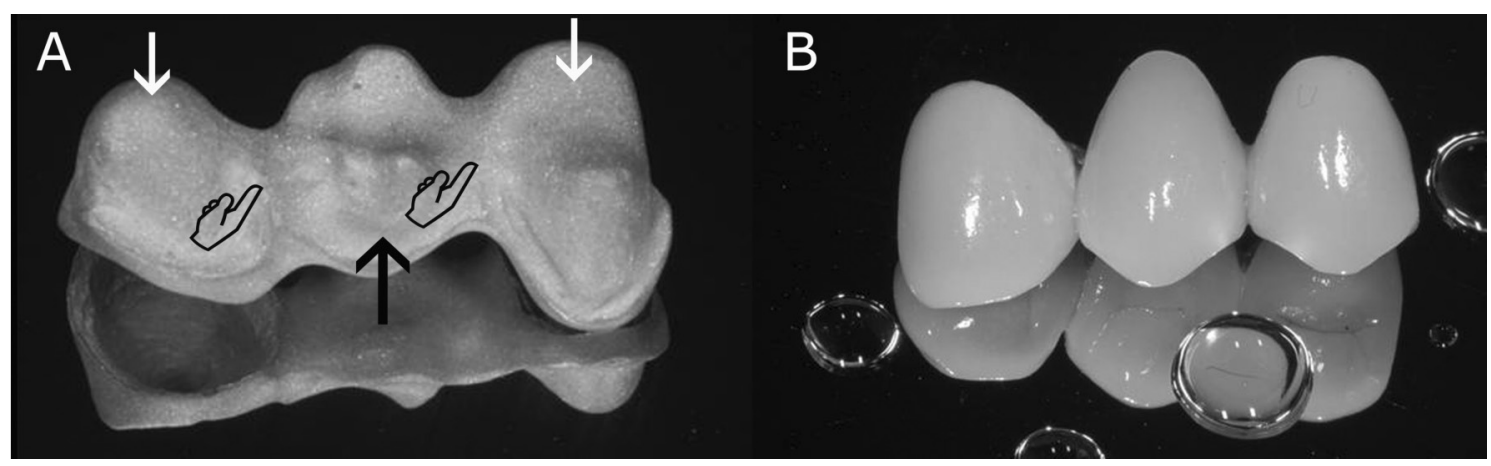

Figura 3: Figura representativa de uma PPF. Em (a) é representada uma infraestrutura metálica onde é possível observar a área de conexão (ponteiras pretas) entre os pilares (setas brancas), devido a ausência de um elemento dentário, o qual será substituído por meio de um pôntico (seta preta). Em (b) observa-se a PPF após a aplicação da porcelana de revestimento. (Caso gentilmente cedido pelo Dr. D. Hiramatsu)

[Figure 3: Representative figure of a Fixed Parcial Denture (FPD). In (a) it is represented a metal framework where it is possible to see the connection area (black tips) between the pillars (white arrows) due to the absence of a missing tooth, which will be replaced by a pontic (black arrow). In (b) it is shown the FPD after application of the porcelain veneer. (Case courtesy of Dr. D. Hiramatsu)]

Tabela I - Sistemas cerâmicos descritos na literatura e áreas dos conectores indicadas, adaptada de [56]. [Table I - Ceramic systems described in the literature and indicated connector areas, adapted from [56].]

\begin{tabular}{|c|c|c|c|c|c|}
\hline Sistema & Fabricante & Material da Infraestrutura & $\begin{array}{l}\text { Altura do conector } \\
\text { recomendada } \\
(\mathrm{mm})\end{array}$ & $\begin{array}{l}\text { Largura do conector } \\
\text { recomendada } \\
(\mathrm{mm})\end{array}$ & $\begin{array}{c}\text { Área da } \\
\text { superfície total } \\
\left(\mathrm{mm}^{2}\right)\end{array}$ \\
\hline $\begin{array}{l}\text { InCeram } \\
\text { Alumina }\end{array}$ & Vita & $\begin{array}{c}\text { Alumina infiltrada por vidro } \\
\text { (somente para PPFs } \\
\text { anteriores) }\end{array}$ & 4 & 3 & 12 \\
\hline $\begin{array}{l}\text { InCeram } \\
\text { Zircônia }\end{array}$ & Vita & $\begin{array}{l}\text { Alumina infiltrada por } \\
\text { vidro com } 35 \% \text { de zircônia } \\
\text { parcialmente estabilizada }\end{array}$ & 4 a 5 & 3 a 4 & 12 a 20 \\
\hline EmpressII & $\begin{array}{c}\text { Ivoclar } \\
\text { Vivadent }\end{array}$ & Dissilicato de lítio & 4 a 5 & 3 a 4 & 12 a 20 \\
\hline $\begin{array}{l}\text { Procera } \\
\text { AllCeram } \\
\text { Bridges }\end{array}$ & $\begin{array}{c}\text { Nobel } \\
\text { Biocare }\end{array}$ & $\begin{array}{l}\text { Alumina de alta pureza } \\
\text { densamente sinterizada }\end{array}$ & 3 & 2 & 6 \\
\hline Cercon & $\begin{array}{l}\text { Dentsply } \\
\text { Ceramco }\end{array}$ & $\begin{array}{l}\text { Zircônia estabilizada } \\
\text { Y-TZP ( sinterizada) }\end{array}$ & - & - & 7 a 11 \\
\hline Precident & $\begin{array}{c}\text { DCS } \\
\text { Dental }\end{array}$ & $\begin{array}{l}\text { Zircônia estabilizada } \\
\text { Y-TZP (sinterizada) }\end{array}$ & 4 & 4 & 16 \\
\hline Lava & 3M ESPE & $\begin{array}{l}\text { Zircônia estabilizada } \\
\text { Y-TZP ( sinterizada) }\end{array}$ & 3 & 3 & 9 \\
\hline
\end{tabular}


é a fratura dos conectores, resultado da propagação de trincas a partir da superfície gengival dos pônticos. Esta região está sujeita a forças de tração, que são forças pobremente suportadas pelas cerâmicas $[43,51,57,58]$. Para minimizar este tipo de falha, os conectores devem ter altura e espessura suficientes para resistir às forças funcionais, respeitando as dimensões mínimas indicadas (Tabela I). Quando o espaço interoclusal é restrito, pode ser difícil construir conectores com altura e largura adequados sem comprometer o espaço de higienização e acomodação da papila gengival [59]. As formas inadequadas dos conectores foram reportadas como fator de início a propagação da trinca [60].

Foram avaliadas próteses fixas feitas de vitrocerâmica IPS Empress II (Ivoclar Vivadent) e encontraram-se três fraturas na área de conexão, justificadas pela deficiência dimensional presente nesta área [41]. Em outro estudo, foram encontrados $90 \%$ de sobrevida em 5 anos de PPF em compósito de alumina infiltrada com vidro (In-Ceram Alumina - Vita Zahnfabrik), sendo que duas próteses na região posterior repondo primeiro molar superior e inferior apresentaram falhas catastróficas com origem na região do conector [61]. Quando avaliadas durante 3 anos, PPFs tanto na região anterior quanto na posterior não apresentaram fraturas na região do conector [62]. Numa revisão sistemática da literatura, foi observado que a perda anual de PPFs é de $6,5 \%$, sendo que para as PPFs de vitrocerâmica e de In-Ceram a fratura da infraestrutura foi a principal complicação, com taxas anuais de $1,88 \%$ e 4,24\%, respectivamente. Entretanto, a fratura da infraestrutura de zircônia é rara, com taxa anual de $0,48 \%$ [31].

Comparada com outros materiais cerâmicos, a zircônia apresenta estabilidade superior da infraestrutura [31] e exibe a combinação de elevada resistência flexural e elevada tenacidade à fratura, associada à propriedade de transformação de fase (tetragonal para monoclínica), além de menor módulo de elasticidade [51, 63, 64]. Estas propriedades, não isoladamente, mas de forma combinada, podem justificar as excelentes taxas de sobrevida para a infraestrutura. Entretanto, a principal causa de complicações mecânicas nas próteses à base de zircônia relaciona-se com a fratura da porcelana de revestimento. Esta pode ser devido à forma (design) deficiente da infraestrutura, o qual não promove suporte adequado à porcelana de cobertura e pode estar associada à concentração de tensão durante a aplicação da porcelana de revestimento. A fratura da porcelana de revestimento para PPFs totalmente cerâmicas é de 13,6\% em 5 anos, estatisticamente diferente da taxa de $2,9 \%$ ao ano observada nas PPFs metalocerâmicas [31]. Melhorar o comportamento dos sistemas cerâmicos à base de zircônia em relação à fratura da porcelana de revestimento é o desafio a ser superado e, dessa forma, ter um sistema totalmente cerâmico, do ponto de vista mecânico, similar ao metalocerâmico.

\section{CONCLUSÃo}

Uma diversidade de sistemas cerâmicos está atualmente disponível no mercado decorrente da busca cada vez maior pela excelência estética na Odontologia. Cada sistema cerâmico apresenta vantagens e desvantagens quando comparados, assim como apresentam indicações específicas e também suas contra-indicações. Através dos estudos clínicos dos diversos sistemas cerâmicos analisados, podese concluir que cabe ao profissional avaliar a real indicação de cada sistema e aplicá-lo na sua clínica diária, sempre considerando que esses novos sistemas possuem tempos de avaliação clínica ainda insuficientes, principalmente quando comparados a estudo clínicos mais longos das tradicionais coroas metalocerâmicas. Sugere-se, portanto, que a utilização desses sistemas seja feito com parcimônia, seguindo estritamente suas indicações, enquanto se espera por resultados de estudos clínicos de longa duração, bem como pelo desenvolvimento de soluções referentes às falhas coesivas das porcelanas de cobertura presentes nos sistemas à base de zircônia.

\section{REFERÊNCIAS}

[1] D. R. Haselton, A. M. Diaz-Arnold, S. L. Hillis, J Prosthet Dent. 83 (2000) 396.

[2] Y. Zhang, B. R. Lawn, J. Biom. Mater. Res. 15, 2 (2005) 388.

[3] W. C. Wagner, T. M. Chu, J. Prosthet Dent. 76 (1996) 140. [4] N. De Jager, M. de Kler, J. M. van der Zel, Dent. Mater. 22, 3 (2006) 234.

[5] M. Guazzato, K. Proos, G. Sara, M. V. Swain, Int. J. Prosthodont. 17, 2 (2004) 142.

[6] J. Tinschert, D. Zwez, R. Marx, K. J. Anusavice, J. Dentistry 28, 7 (2000) 529.

[7] J. R. Kelly, I. Nishimura, S. D. Campbell, J. Prosthet Dent. 75, 1 (1996) 18.

[8] R. A. Giordano, Compend Contin. Educ. Dent. 17, 8 (1996) 779.

[9] J. A. Sorensen, M. Cruz, W. T. Mito, O. Raffeiner, H. R. Meredith, H. P. Foser, Pract Proced Aesthet Dent. 11, 1 (1999) 95.

[10] M. J. Heffernan, S. A. Aquilino, A. M. Diaz-Arnold, D. R. Haselton, C. M. Stanford, M. A. Vargas, J. Prosthet. Dent. 88, 1 (2002) 4.

[11] M. Albakry, M. Guazzato, M. V. Swain, J. Dent. 31, 3 (2003) 181.

[12] M. V. Swain, Mater. Forum 13 (1989) 237.

[13] T. Kampe, G. E. Carlsson, H. Hannerz, T. Haraldson, Acta Odontol. Scand. 45, 1 (1987) 25.

[14] D. C. Cosme, S. M. Baldisserotto, S. A. Canabarro, R. S. Shinkai, J. Prosthet. Dent. 18, 4 (2005) 328.

[15] A. J. Raigrodski, J. Prosthet. Dent. 92, 6 (2004) 557.

[16] P. F. Cesar, H. N. Yoshimura, W. G. Miranda Junior, C. Y. Okada, J. Dent. 33, 9 (2005) 721.

[17] J. R. Mackert, C. M. Jr., Russell, Int. J. Prosthodont. 9, 3 (1996) 261.

[18] M. Guazzato, M. Albakry, S. P. Ringer, M. V. Swain, Dent. Mater. 20, 5 (2004) 441.

[19] L. C. Sobrinho, M. J. Cattell, J. C. Knowles, J. Mater. 
Sci. 9, 10 (1998) 555.

[20] Vivadent I. [http://www.ivoclarvivadent.com]

[21] G. Mitov, U. Lohbauer, M. A. Rabbo, A. Petschelt, P Pospiech, Dent. Mater. 24, 2 (2008) 267.

[22] M. L. Myers, J. W. Ergle, C. W. Fairhurst, R. D. Ringle. Int. J. Prosthodont. 7, 6 (1994) 549.

[23] H. Attaoui, M. S. Lersim, J. Chevalier, G. Fantozzi. J. Am. Ceram. Soc. 88, 1 (2005) 172.

[24] R. Morena, G. M. Beaudreau, P. E. Lockwood, A. L. Evans, C. W. Fairhurst, J. Dental Res. 65, 7 (1986) 993.

[25] D. Jones, H. J. Wilson, J. Oral Rehabil. 2 (1975) 379.

[26] D. W. Jones, P. A. Jones, H. J. Wilson, J. Dent. 1, 2 (1972) 85.

[27] P. F. Cesar, C. C. Gonzaga, W. G. Miranda Jr., H. N. Yoshimura, J. Biomed. Mater. Res. B - Appl. Biomater. 83B (2007) 538.

[28] W. E. C. Creyke, Trans. Br. Ceram. Soc. 67 (1968) 339.

[29] K. J. Anusavice, B. Hojjatie, J. Dent. Res. 70, 6 (1991) 1009.

[30] J. W. McLean, T. H. Hughes, Br. Dent. J. 119, 6 (1965) 251.

[31] I. Sailer, B. E. Pjetursson, M. Zwahlen, C. H. Hammerle, Clin. Oral Implants Res. 18 (2007) 86.

[32] J. Chevalier, Biomaterials 27, 4 (2006) 535.

[33] P. F. Manicone, P. Rossi Iommetti, L. Raffaelli, J. Dent. 35, 11 (2007) 819.

[34] S. Lawson, J. Eur. Ceram. Soc. 15 (1995) 484.

[35] K. Kobayashi, H. Kuwajima, T. Masaki, Solid State Ionics 3/4 (1981) 489.

[36] J. Wang, R. Stevens, Ceram. Int. 15 (1989) 15.

[37] M. Yoshimura, Am. Ceram. Soc. Bull. 67, 12 (1988) 1950.

[38] J. Tinschert, K. A. Schulze, G. Natt, P. Latzke, N. Heussen, H. Spiekermann, Int. J. Prosthodont. 21, 3 (2008) 217.

[39] J. W. Callister, An Introduction to Materials Science and Engineering, John Wiley \& Sons Inc., New York, EUA (2003) p. 129.

[40] B. E. Pjetursson, I. Sailer, M. Zwahlen, C. H. Hammerle, Clin. Oral Implants Res. 18 (2007) 73.

[41] P. Marquardt, J. R. Strub, Quintessence Int. 37, 4 (2006) 253.

[42] H. De Backer, G. Van Maele, N. De Moor, L. Van den Berghe, J. De Boever, Int. J. Prosthodont. 19, 2 (2006) 136.

[43] K. J. Anusavice, R. W. Phillips RW, Phillips' science of dental materials, W. B. Saunders Co., London, UK (2003) p. 345 .

[44] B. R. Lawn, Y. Deng, V. P. Thompson. J. Prosthet. Dent. 86, 5 (2001) 495.

[45] J. R. Kelly, Dent. Clin. North Am. 48, 2 (2004) 513.

[46] H. W. Wiskott, J. I. Nicholls, U. C. Belser, Int. J. Prosthodont. 8, 2 (1995) 105.

[47] P. F. Cesar, H. N. Yoshimura, W. G. Miranda Jr., C. L. Miyazaki, L. M. Muta, L. E. Rodrigues Filho, J. Biomed. Mater. Res. B - Appl. Biomater. 78, 2 (2006) 265.

[48] J. R. Kelly, S. D. Campbell, H. K. Bowen, J. Prosthet. Dent. 62, 5 (1989) 536.

[49] B. R. Lawn, Y. Deng, I. K. Lloyd, M. N. Janal, E. D. Rekow, V. P. Thompson. J. Dent. Res. 81, 6 (2002) 433.

[50] D. Rekow, Y. Zhang, V. Thompson, Compend Contin. Educ. Dent. 28, 7 (2007) 362.

[51] B. Kim, Y. Zhang, M. Pines, V. P. Thompson, J. Dent. Res. 86, 2 (2007) 142.

[52] E. D. Rekow, M. Harsono, M. Janal, V. P. Thompson, G. Zhang G, Dent. Mater. 22, 2 (2006) 125.

[53] A. Bindl, W. H. Mormann, Int. J. Prosthodont. 15, 5 (2002) 451.

[54] E. A. McLaren, S. N. White, J. Prosthet. Dent. 83, 2 (2000) 216.

[55] T. Takeda, K. Ishigami, A. Shimada, K. Ohki, Int. J. Prosthodont. 9, 2 (1996) 197.

[56] A. J. Raigrodski, A. M. Saltzer, Pract. Proced. Aesthet. Dent. 14, 5 (2002) 411.

[57] J. R. Kelly, J. Prosthet. Dent. 81, 6 (1999) 652.

[58] J. R.Kelly, J. A. Tesk, J. A. Sorensen, J. Dent. Res. 74, 6 (1995) 1253.

[59] C. M. Becker, W. B. Kaldahl, J. Prosthet. Dent. 45, 3 (1981) 268.

[60] P. Pospiech, P. Rammelsberg, G. Goldhofer, W. Gernet, Eur. J. Oral Sci. 104, 4 (1996) 390.

[61] P. Vult von Steyern, O. Jonsson, K. Nilner, Int. J. Prosthodont. 14 (2001) 379.

[62] A. Piwowarczyk, H. C. Lauer, J. A. Sorensen, Oper. Dent. 30, 3 (2005) 382.

[63] H. J. Conrad, W. J. Seong, I. J. Pesun, J. Prosthet. Dent. 98, 5 (2007) 389.

[64] A. J. Raigrodski, G. J. Chiche, N. Potiket, J. L. Hochstedler, S. E. Mohamed, S. Billiot, D. E. Mercante, J. Prosthet. Dent. 96, 4 (2006) 237.

(Rec. 08/07/2009, Rev. 07/10/2009, Ac. 16/11/2009) 